\title{
Mutagenic Oligonucleotide-directed PCR Amplification (Mod-PCR): An Efficient Method for Generating Random Base Substitution Mutations in a DNA Sequence Element
}

\author{
Lillian W. Chiang, ${ }^{1,2}$ Iulia Kovari, ${ }^{1}$ and Martha M. Howe ${ }^{1}$ \\ ${ }^{1}$ Department of Microbiology and Immunology, The University of Tennessee-Memphis, Memphis, Tennessee 38163; \\ ${ }^{2}$ Department of Bacteriology, University of Wisconsin-Madison, Madison, WI 53706
}

Saturation mutagenesis is one approach for determining the contributions of individual base pairs to the structure and function of defined DNA sequence elements. In this paper, we describe a novel method for saturation mutagenesis involving PCR amplification with degenerate synthetic oligonucleotides as primers. The degeneracy is confined to a specific target within the primer by mixing a low percentage of the three non-wild type (non-WT) nucleotide precursors with $W T$ at specific positions during primer synthesis. PCR amplification of WT template DNA with the degenerate primer and an opposing WT primer, followed by subsequent cloning using restriction sites designed into the primers, results in recovery of a population of randomly mutated products. Since primers with multiple mutations hybridize less efficiently to WT template DNA during PCR amplification, the recovery of mutants with multiple base changes is greatly reduced. The efficient generation of random point mutations with this method allows the construction of separate mutant populations, each mutagenized over a different portion of the DNA sequence element. If a phenotypic assay is available, these populations can be screened directly to define those regions within the element that are important for activity. Only those populations containing mutations in the important regions require further characterization by DNA sequence analysis.

$\Delta$ complete dissection of a DNA sequence element, such as the binding site of a protein previously defined by footprinting, includes the isolation of every possible single point mutation within the sequence of interest and the analysis of the resulting phenotypic effects. Current methods for generating such mutations include site-directed mutagenesis by specific synthetic oligonucleotides, ${ }^{(1)}$ random mutagenesis with degenerate oligonucleotides, ${ }^{(2-4)}$ and mutagenesis during PCR by Taq DNA polymerase-mediated misincorporation. ${ }^{(5-6)}$

The use of separate synthetic oligonucleotides, each with a different defined base substitution, is ideal for the isolation of a few mutants with specific desired base changes, such as the creation of a restriction site or alteration of a specific amino acid codon. However, it is an expensive and labor-intensive process when applied to the saturation mutagenesis of even a small, e.g., 10-base, sequence.

Mutagenesis with degenerate oligonucleotides containing randomly incorporated substitutions requires considerably fewer syntheses. However, cloning of the mutant oligonucleotides requires the generation of closely spaced restriction sites within the element or the syn- thesis of long oligonucleotides to span the region between preexisting restriction sites. ${ }^{(3-4)}$

Mutagenesis during PCR occurs naturally at a low frequency of $\sim 0.2 \%$ per nucleotide $^{(5)}$ and can be increased to $\sim 2 \%$ under suboptimal reaction conditions ${ }^{(6)}$; therefore, it is inefficient for the saturation mutagenesis of a small 10-bp element.

In this paper, we describe a mutagenesis procedure that involves PCR amplification with degenerate oligonucleotide primers. This method reduces the cost of saturation mutagenesis by reducing the length of the oligonucleotides synthesized. It also allows for efficient mutant generation while biasing against the recovery of mutants with multiple mutations. It is, therefore, particularly effective for defining the role of specific bases in DNA sequence elements, by identifying single base changes that cause a significant alteration in element function.

As with direct cloning of a degenerate oligonucleotide, this method can be easily applied to mutagenesis of a larger element, for example, a prokaryotic promoter. Prior to saturation mutagenesis, the boundaries of such elements are often defined by deletion analysis, identifying the element as a 50- to 150-bp segment, only portions of which may be important for function. Important and unimportant regions can be identified quickly by dividing the large element into smaller regions and independently saturating each small region with ran- 


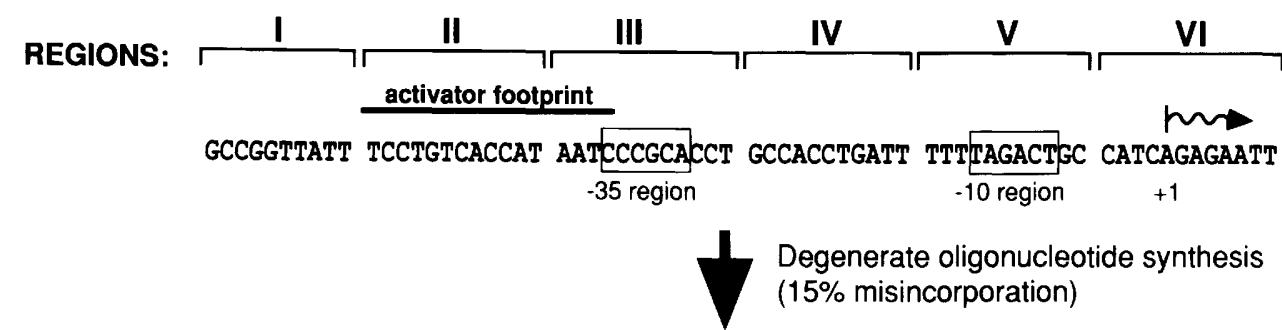

POOLS OF DEGENERATE OLIGONUCLEOTIDES:

Region I primers:
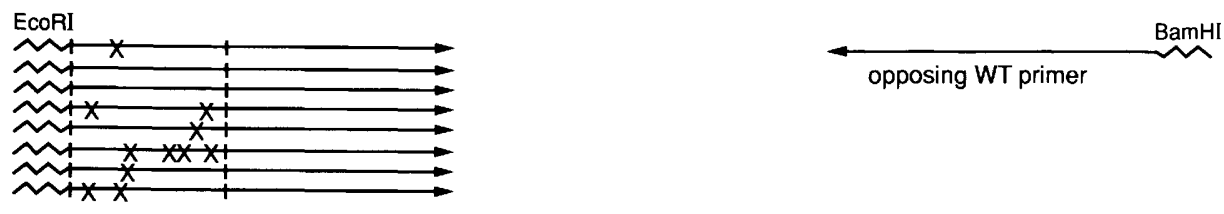

Region $V$ primers:
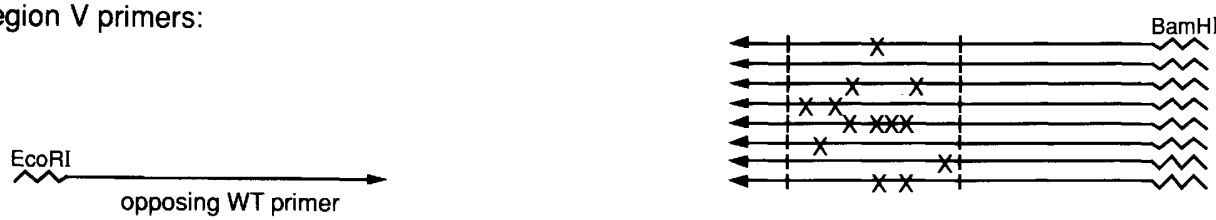

Region 1

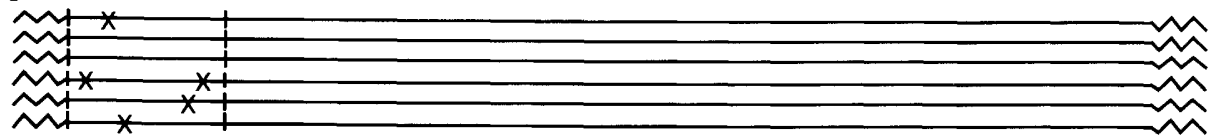

Region V

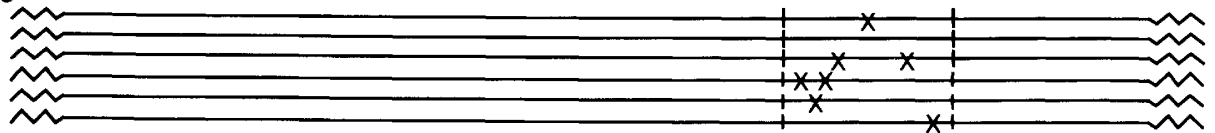

Clone and screen mutant populations

by phenotypic assays

EXPECTED REGIONAL PHENOTYPES:

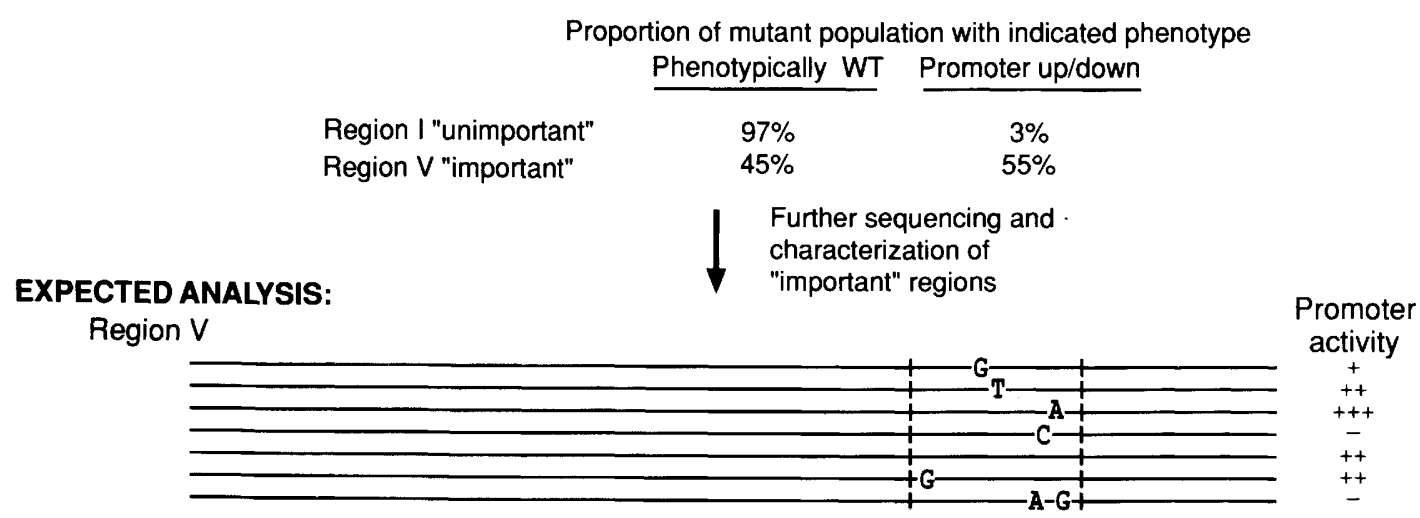

FIGURE 1 Strategy for the genetic dissection of a DNA sequence element, the lys promoter of bacteriophage Mu, by mutagenic oligonucleotidedirected PCR. 
dom point mutations. The number and degree of phenotypic changes exhibited by each regionally distinct population of mutants indicate the importance of each region to activity of the whole element. Such a phenotypic screen significantly reduces the work involved in characterizing the mutants because those generated in unimportant regions need not be sequenced or characterized further. Sequence analysis of all mutations in the important regions, regardless of phenotype, will define the relative contribution of individual base pairs to the activity of the element.

To be effective for identifying important and unimportant regions, the mutagenesis method must allow the isolation of populations that are randomly and efficiently mutagenized in the targeted region and wild-type in the remaining nontargeted bases of the element. To test the method, we have applied it separately to two regions within a 68-bp promoter. Mutagenesis of each region involved two steps: (1) Base substitution mutations within the targeted region were incorporated during synthesis of a degenerate oligonucleotide to be used as a primer for PCR. (2) PCR amplification with the degenerate primer and an opposing WT primer led to incorporation of the mutant primer and restoration of the remaining WT bases in the 68-bp element. DNA sequence analysis of the mutants demonstrated that this technique is an efficient method for the generation of random point mutations within the targeted DNA segments.

\section{MATERIALS AND METHODS}

\section{Oligonucleotide Synthesis}

Oligomers were synthesized on an Applied Biosystems DNA synthesizer (model 380B) using the phosphite triester method. ${ }^{(7,8)} \beta$-Cyanoethyl phosphoramidites, diluted to $0.1 \mathrm{M}$ in acetonitrile under nitrogen gas to prevent $\mathrm{O}_{2}$ inactivation, were 1.0-gram stocks from Applied Biosystems (A, \#400326, G, \#400327, C, \#400328, and T, \#400329). After, detachment and removal of protecting groups in 30\% ammonium hydroxide at $55^{\circ} \mathrm{C}$ overnight, the oligomers were dried down, resuspended in $200 \mu \mathrm{l}$ of $\mathrm{H}_{2} \mathrm{O}$, extracted three times with an equal volume of $\mathrm{H}_{2} \mathrm{O}$-saturated ether, ${ }^{(9)}$ ethanol precipitated, ${ }^{(9)}$ and resuspended in $100 \mu \mathrm{l}$ of $\mathrm{H}_{2} \mathrm{O}$. Degeneracy was introduced to the positions targeted for $\mathrm{mu}$ tagenesis by simultaneous delivery of nucleotide from two bottles, one with the WT nucleotide and a second containing an equimolar mix of all four nucleotides (Applied Biosystems, A/G/C/T cyanoethyl phosphoramidite, $250 \mathrm{mg}$, \#400334). The latter bottle was diluted to achieve a total phosphoramidite concentration of $0.025 \mathrm{M}$, i.e., $6.25 \mathrm{~mm}$ of each of the nucleotides, A, G, C, and T. Thus, for each targeted nucleotide, synthesis was accomplished with a mixture of $85 \%$ WT and $15 \%$ non-WT nucleotides, resulting in a misincorporation rate of 0.15 per nucleotide. The four oligomers synthesized were ATGGAATTCCCGCCGGTTATTTCCTGTCAC, ATGAATTCCCgccggttattTCCTGTCACC, CGGGATCCCCAATTCTCTGATGgcagtctaaaaAATCAG, and ACGGGATCCCCAATTCTCTGATGGCAGTCTA, where a lower-case letter indicates a position targeted for mutagenesis.

\section{Preparation of PCR Templates}

Template DNA was isolated from Escherichia coli K-12 strain MH3016 ( $\mathrm{F}^{-}$thyA malT::[$\left.\left[\begin{array}{lll}\mathrm{Mu} & \text { cts62 }\end{array}\right]\left[\begin{array}{lll}\mathrm{P} 1^{\mathrm{R}} & \mathrm{P} 2^{\mathrm{R}} & \mathrm{Mu}^{\mathrm{R}}\end{array}\right]\right) .{ }^{(10)}$ Briefly, MH3016 was heat induced to stimulate $\mathrm{Mu}$ replication and thereby increase the copy number of Mu DNA containing the promoter region. Approximately $10-15 \mathrm{~min}$ before lysis, total cellular DNA was isolated by a modified miniprep procedure. ${ }^{(11,12)}$ For PCR, 100 $\mathrm{ng}$ of the isolated template was denatured in a $10-\mu$ l volume of $0.2 \mathrm{M} \mathrm{NaOH}$ for $15 \mathrm{~min}$ at room temperature, followed by 10 -fold dilution into $\mathrm{H}_{2} \mathrm{O}$. One nanogram of denatured template was used immediately in each PCR amplification reaction.

\section{Amplification Conditions}

PCR amplifications were carried out in $100-\mu 1$ reactions containing Taq DNA polymerase reaction buffer $(10 \mathrm{~mm}$ Tris$\mathrm{HCl}, \mathrm{pH} 8.3,50 \mathrm{mM} \mathrm{KCl}$, and $0.001 \% \mathrm{wt} /$ vol gelatin), $1.5-10 \mathrm{~mm} \mathrm{MgCl}_{2}$ maxi-

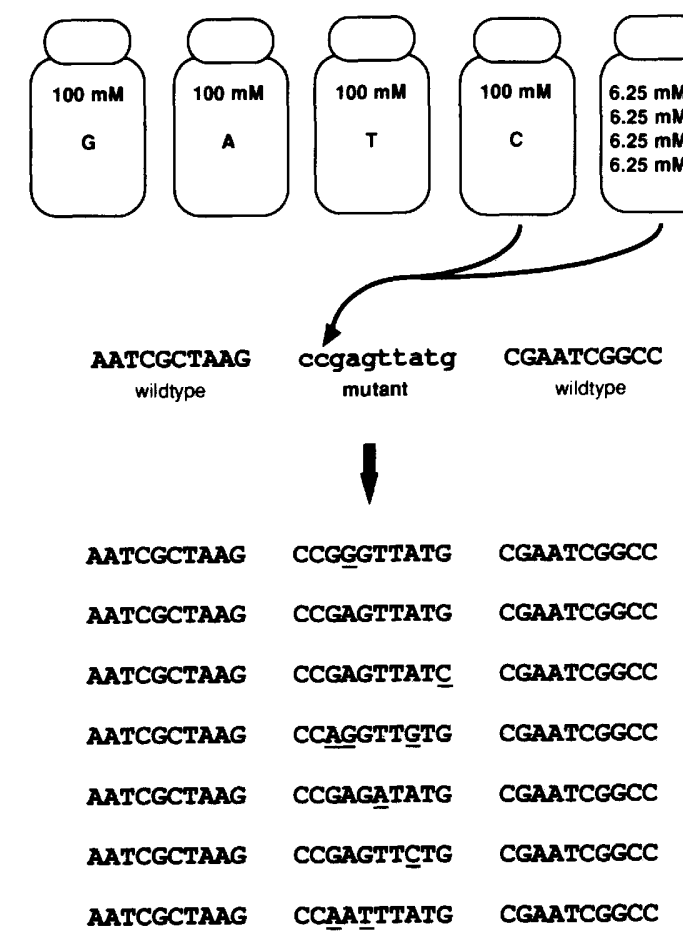

Pool of oligonucleotides with random base substitutions at $\sim 15 \%$ misincorporation

FIGURE 2 Mutagenic oligonucleotide synthesis. Symbols within bottles indicate concentrations of specific nucleotide precursors used for synthesis. In the nucleotide sequence lowercase a, g, c, and $t$ indicate positions targeted for misincorporation; for these positions, nucleotide precursors from two bottles are added simultaneously. In the resulting products $A, G, C$, and $T$ indicate the nucleotide present; underlined nucleotides differ from the WT nucleotides. 
mized for specificity and recovery of amplified product ( $1.5 \mathrm{~mm}$ for Region I, and $6 \mathrm{mM}$ for Region V), $200 \mu \mathrm{M}$ of each dNTP, $1 \mu \mathrm{M}$ of each of the opposing primers, 2.5 units of AmpliTaq polymerase (Perkin-Elmer Cetus), and $1 \mathrm{ng}$ of denatured template (as described above). After overlaying with $50 \mu$ l of mineral oil, the reactions were subjected to 35 cycles of PCR: $1 \mathrm{~min}$ at $94^{\circ} \mathrm{C}$ to denature, $30 \mathrm{sec}$ at $45^{\circ} \mathrm{C}$ to anneal, and $2 \mathrm{~min}$ at $72^{\circ} \mathrm{C}$ to extend. The amplification products were extracted once with $\mathrm{CH}_{3} \mathrm{Cl}$ (24: $1 \mathrm{CH}_{3} \mathrm{Cl} /$ isoamyl alcohol) to remove the mineral oil, and $5 \mu \mathrm{l}$ was subjected to electrophoresis in a $4 \% \quad 3: 1$ NuSieve (FMC Bioproducts) minigel to monitor the amount of product recovered.

\section{DNA Recovery and Cloning}

The remaining PCR product $(\sim 95 \mu \mathrm{l}$ after minigel analysis) was extracted once with an equal volume of phenol- $\mathrm{CH}_{3} \mathrm{Cl}$ (saturated with Tris- $\mathrm{HCl}, \mathrm{pH} 8.0)^{(9)}$ and ethanol precipitated. The pellet was directly resuspended in $10 \mu \mathrm{l}$ of a modified EcoRI/BamHI restriction buffer $(0.15 \%$ Triton X-100, $100 \mathrm{~mm} \mathrm{NaCl}, 50 \mathrm{~mm}$ Tris$\mathrm{HCl}, \mathrm{pH} 7.5,10 \mathrm{~mm} \beta$-mercaptoethanol, and $\left.5 \mathrm{~mm} \mathrm{MgCl}_{2}\right)$, containing 5 units of BamHI (New England BioLabs, Inc.), 2 units of EcoRI (New England BioLabs, Inc.), and $0.1 \mathrm{mg} / \mathrm{ml}$ bovine serum albumin (diluted in $\mathrm{H}_{2} \mathrm{O}$ from New England BioLabs, Inc., $10 \mathrm{mg} / \mathrm{ml}$ stock). The digestions were carried out for $2 \mathrm{hr}$ at $37^{\circ} \mathrm{C}$ or overnight at room temperature. The products were purified in a $4 \%$ NuSieve agarose gel according to FMC Bioproducts' protocols. Ligations were carried out in the melted NuSieve agarose as recommended by FMC Bioproducts with pLC1 vector which had been previously digested with EcoRI and BamHI, treated with calf intestine alkaline phosphatase (Boehringer Mannheim), and gel purified. Plasmid pLC1 is a lacY derivative of the promoter cloning vector, $\mathrm{pRS} 415,{ }^{(13)}$ generated by deletion of the SnaBI fragment in lacY. The ligation mixtures were transformed into MH8624 (spro-lac, trp $\Delta \mathrm{ED} 24$, recA, pLC3) made competent by $\mathrm{CaCl}_{2}$ treatment $^{(9)}$ and stored frozen. Plasmid pLC3 is a monomeric derivative of $\mathrm{pWM} 13^{(14)}$ with the lacl $^{\mathrm{Q}}$ cassette from pMJR1560 ${ }^{(15)}$ cloned into the Taql methylase-insensitive HincII site of pWM13; this plasmid was present to provide an activator necessary for the phenotypic assay of promoter activity.

\section{Sequence Analysis}

Double-stranded sequencing templates of the mutated promoter clones were prepared by a modified Holmes and Quigley miniprep method involving lysis by boiling. ${ }^{(12,16)}$ The minipreps were alkali denatured in $100 \mu \mathrm{l}$ of $0.2 \mathrm{M} \mathrm{NaOH}$ for $30 \mathrm{~min}$ at $37^{\circ} \mathrm{C}$, neutralized with 40 $\mu l$ of $3 \mathrm{~m}$ sodium acetate $(\mathrm{pH} 4.8)$, and ethanol precipitated before being subjected to standard US Biochemicals Sequenase sequencing methods using deoxyadenosine $5^{\prime}$-[ $\alpha$-thio-] triphosphate, $\left[{ }^{35} \mathrm{~S}\right]$ - (NEN Research Products) as label. Manganese buffer was added to the sequencing reactions according to US Biochemicals Sequenase protocols to obtain sequences close to the primer (CAGGAATTGGGGATCGG), which was homologous to pLC1 upstream of the cloning site. The sequence was visualized by $6 \%$ PAGE in the presence of $8 \mathrm{M}$ urea followed by autoradiography. ${ }^{(9)}$

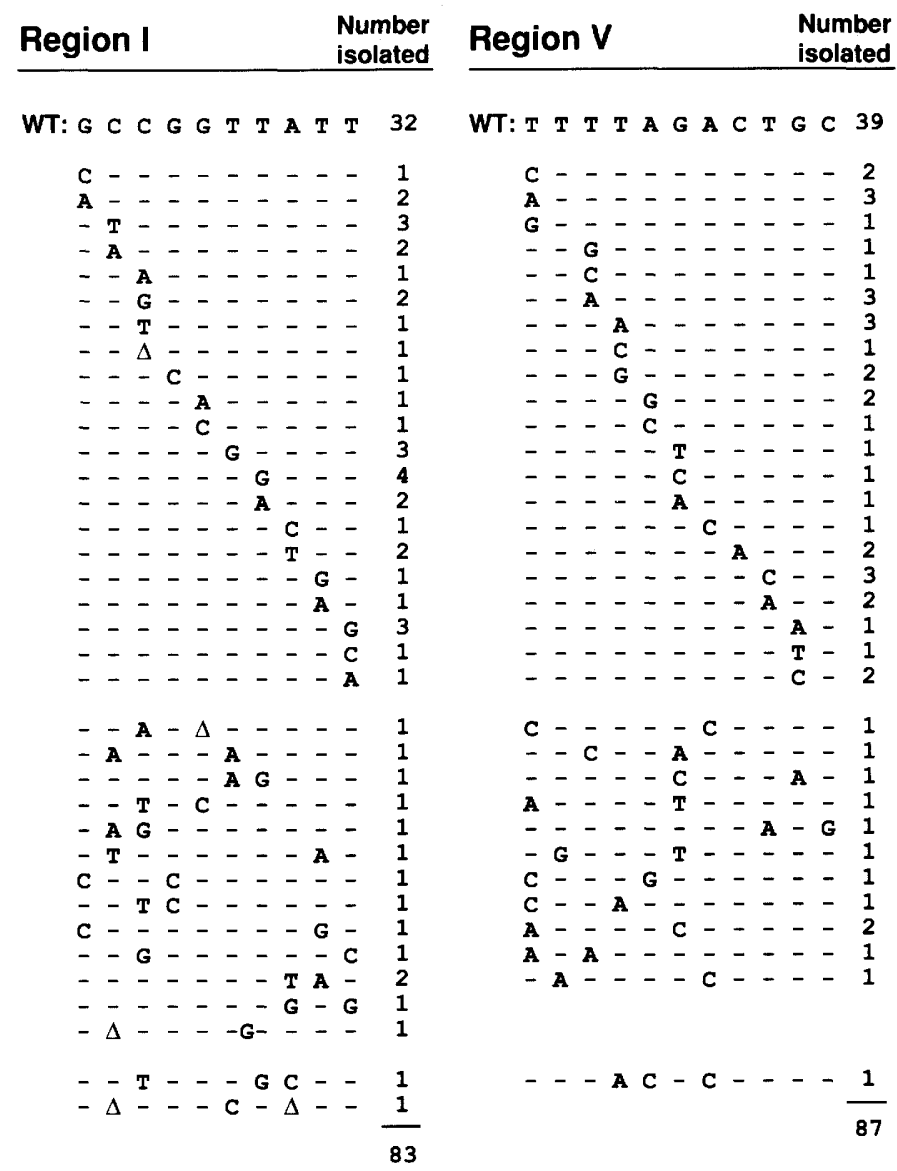

FIGURE 3 Distribution of base changes recovered in Regions I and V of $P_{l y s}$. The WT DNA sequence of the region targeted for mutagenesis is shown at the top. The number of times a specific sequence was found is indicated on the right. For the mutants, only the mutant nucleotides are indicated; $\Delta$ represents a deleted nucleotide; the $G$ in the last Region I double mutant represents an inserted $\mathrm{G}$.

\section{RESULTS}

As a test of the method, mutagenesis by degenerate oligonucleotide-primed PCR amplification (Mod-PCR for mutagenic oligonucleotide-directed PCR) was applied to two regions within the bacteriophage $\mathrm{Mu}$ late promoter, $\mathrm{P}_{\text {lys }}$. First, $\mathrm{P}_{\text {lys }}$ was divided into appropriate target regions for mutagenesis and screening of resulting phenotypic changes. Next, a degenerate primer was synthesized for each target region and used in an independent PCR amplification with an opposing WT primer. Finally, the amplification products were cloned, assayed for promoter activity, and sequenced to determine the efficacy of the mutagenesis method.

\section{Regional Mutagenesis Strategy}

Figure 1 illustrates the hypothetical application of regional mutagenesis and 
phenotypic screening to $\mathrm{P}_{\text {lys }}$. The 68-bp $\mathrm{P}_{\text {lys }}$ promoter would be divided into six 10- to 12-bp regions such that groups of bases expected to be important would be in one region and others predicted to be less important would be in a different region. Mutant populations for each region would be generated by Mod-PCR and cloned into a promoterless lac $Z$ expression vector so that an indicator plate could be used to assay promoter activity. The proportion of clones with WT and non-WT phenotypes would indicate the relative contribution of each region to promoter activity. Region $\mathrm{V}$ represents a region important for activity; many of the clones in the Region $\mathrm{V}$ population would exhibit an up or down promoter phenotype. Region I represents a less important region in which the majority of mutants would be phenotypically WT. Many clones in the important Region $\mathrm{V}$ population would be sequenced and characterized, regardless of phenotype. For the unimportant Region I, only the clones with altered phenotype would be analyzed further; however, a few phenotypically WT clones, perhaps 10 , would be sequenced to demonstrate that phenotypically silent mutations were present. Thus, the application of ModPCR to specific regions of an element could greatly reduce the number of mutants that need to be sequenced to determine the bases required for activity.

In this paper, we will present the results of sequence analysis of mutants in both Regions I and V (even though Region I is "unimportant") because they represent independent tests of the ModPCR method using primers that were synthesized with separately prepared nucleotide substrates. The phenotypic properties of the regionally mutagenized populations and of specific mutants will be presented in a subsequent paper.

\section{Oligonucleotide Synthesis and PCR}

An oligomer of -30 nucleotides encompassing each targeted region was synthesized with an automated DNA synthesizer. During synthesis of the WT portion of the oligomer, the appropriate WT nucleotide was added to the synthesis chamber from one bottle. When a nucleotide targeted for mutagenesis was reached, equal volumes were added simultaneously from two different bottles: one contained the WT nucleotide; the other contained an equimolar mixture of all four nucleotides, each at $1 / 16$ th the concentration of nucleotide in the WT bottle (Fig. 2). Thus, the resulting nucleotide mixture in the synthesis chamber contained 5\% each of the three non-WT nucleotides. For a 10-bp mutagenized region, this should result in an average of 1.5 misincorporations (non-WT nucleotides) per oligomer, a level that maximizes the proportion of one- and two- base substitutions. This five-bottle synthesis minimizes nonrandom substitution that can result with the alternate method of preparing separately spiked $A$, $\mathrm{G}, \mathrm{C}$, and $\mathrm{T}$ mixes.

The degenerate primers were designed to contain an overall $\mathrm{G}+\mathrm{C}$ content of $\sim 50 \%$, at least four WT nucleotides on the $3^{\prime}$ end to ensure efficient priming and, where appropriate, a restriction site on the $5^{\prime}$ end to allow direct cloning into the assay vector. The re- maining WT bases in the 68-bp promoter were restored by PCR amplification using a WT primer for the opposing end (Fig. 1). After EcoRI and BamHI digestion, the PCR products were cloned into pLC1, the promoterless lacZ expression vector, for phenotypic assay and sequence analysis.

\section{Randomness and Efficiency of Mod-PCR}

Sequence analysis of clones from the Region I and Region V mutagenized populations revealed that only 2 of 170 clones contained a mutation outside the targeted regions (a 1-bp deletion and a 1-bp substitution), confirming the low mutagenic activity of PCR itself $(\sim 0.08 \%$ per base in our hands for $>25,000$ bases sequenced), and demonstrating the fidelity of restoration of WT sequences

TABLE 1 Assessment of Degree of Randomness of Mutagenesis Method

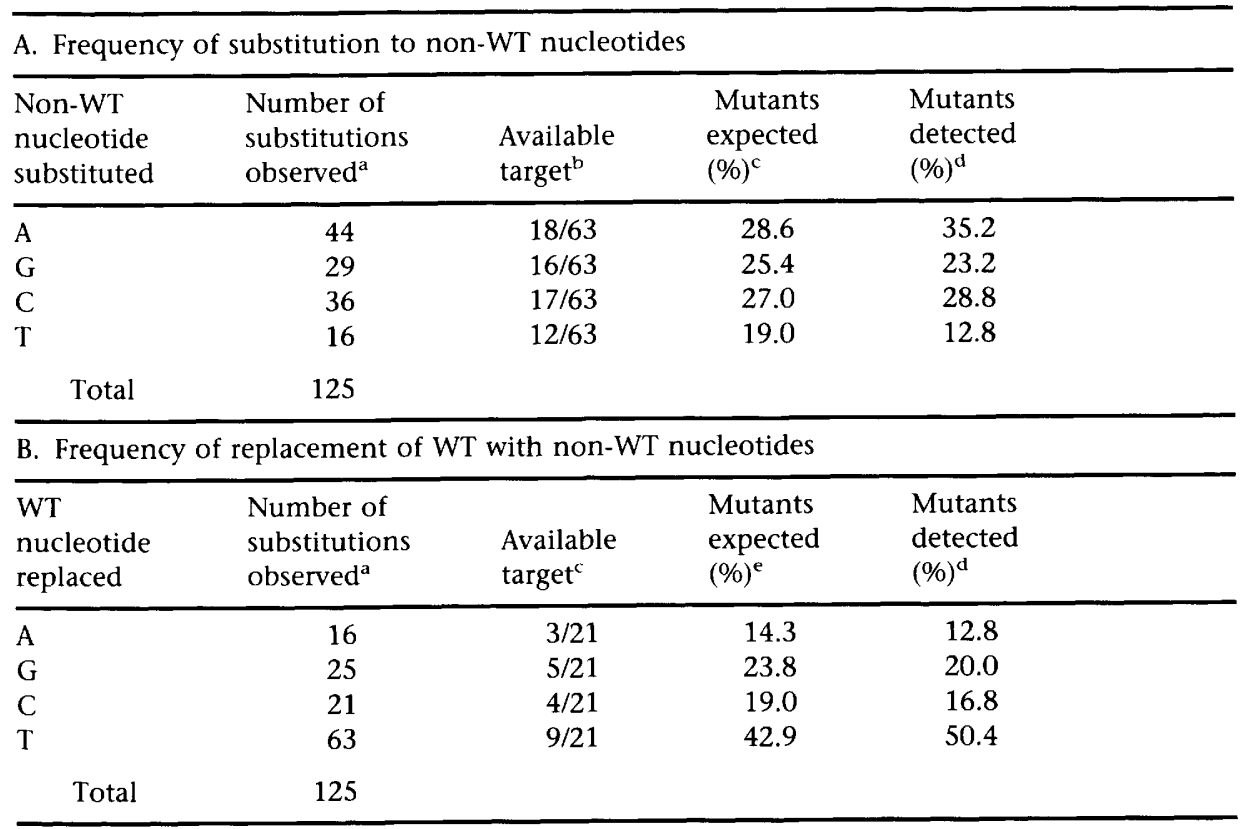

aThe number of substitutions observed for each class were tallied for substitution to specific non-WT nucleotides (A) and replacement of specific WT nucleotides (B), for all nucleotide changes shown in Fig. 2 except deletions and insertions.

${ }^{b}$ The numerator of the available target for substitution to non-WT nucleotides was determined by the number of positions targeted for mutagenesis in Regions I and V which were not the nucleotide indicated. The denominator was defined by the total number of possible substitutions. In other words, because 21 positions were targeted for mutagenesis in Regions I and V, and at each position 3 non-WT possibilities existed, the total number of possible substitutions was $21 \times 3=63$.

'The expected percentage of each mutant class was calculated from the fraction of available target.

${ }^{\mathrm{d}}$ The detected percentage was the number of substitutions observed in each class divided by the total number of substitutions (125).

'The available target for replacement of WT nucleotide was the number of WT positions of the indicated nucleotide among 21 total. 
TABLE 2 Bias Against Multiple Base Substitutions by Mod-PCR Mutagenesis

\begin{tabular}{lccc}
\hline A. Region I & & & \\
\hline Number of changes & ${\text { Expected }(\%)^{\mathrm{a}}}$ & ${\text { Observed }(\%)^{\mathrm{b}}}$ & ${\text { Recovery }(\%)^{\mathrm{c}}}$ \\
\hline 0 & 19.7 & 38.6 & 100 \\
1 & 34.7 & 42.2 & 62.1 \\
2 & 27.6 & 16.9 & 31.2 \\
More & 18.0 & 2.4 & 6.8 \\
\hline
\end{tabular}

B. Region $\mathrm{V}$

\begin{tabular}{lccc}
\hline Number of changes & ${\text { Expected }(\%)^{\mathrm{a}}}^{\mathrm{a}}$ & ${\text { Observed }(\%)^{\mathrm{b}}}$ & ${\text { Recovery }(\%)^{\mathrm{c}}}^{\mathrm{c}}$ \\
\hline 0 & 16.7 & 44.8 & 100 \\
1 & 32.5 & 40.2 & 46.1 \\
2 & 28.7 & 13.8 & 17.9 \\
More & 22.1 & 1.2 & 2.0 \\
\hline
\end{tabular}

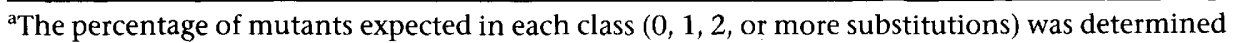
by the binomial theorem with a misincorporation rate of 0.15 , and a target size of $10 \mathrm{bp}$ for Region I and $11 \mathrm{bp}$ for Region V.

${ }^{\mathrm{b}}$ The percentage of mutants observed in each class was determined by sequence analysis of 83 and 87 mutants in Region I and V, respectively.

'The percent recovery of mutants was calculated relative to $100 \%$ recovery of the 0 change (WT) class. within regions not targeted for mutagenesis.

Figure 3 shows the distribution of base changes recovered among 170 clones resulting from Mod-PCR mutagenesis. The isolation of at least one base change at every position targeted for mutagenesis was an encouraging sign that the mutagenesis was random. In Table 1 we more systematically investigated the degree of randomness by first calculating the expected distribution of specific substitutions based on the target size of each base within the mutagenized sequence. For example, the available target for substitution to $\mathrm{A}$ is given by 18 positions which are not A within the 21 bp targeted for mutagenesis (10 bp of Region I plus 11 bp of Region V). Since the total number of possible non-WT substitutions is 3 non-WT possibilities per position $(21 \times 3=63)$, the expected fraction of mutations to A should be 18 divided by 63 , or $28.6 \%$ (Table $1 \mathrm{~A}$ ). A similar calculation was done to define the expected frequency of mutation from a specific WT nucleotide to any of the non-WT nucleotides (Table 1B). Because the detected distribution of base changes approached that expected for nonbiased misincorporation (Table 1), substitution by non-WT nucleotides and sentially the same for $A, G, C$, or $T$.

As expected, due to the requirement for primer hybridization to target DNA replacement of WT nucleotides was es- during PCR, the number of mutants recovered with multiple base substitutions (more than two substitutions) was considerably less than that predicted by the binomial theorem; only $2.4 \%$ and $1.2 \%$ tively, contained more than two substitutions (Table 2). In fact, comparison of the observed distribution to that expected indicates that recovery of the WT class ( 0 base changes) was significantly favored. The percent recovery by ModPCR of base substitutions theoretically present in the degenerate primer pool was compared to that of the WT class. Relative to WT (100\%), one-base substitutions were recovered at $62 \%$ and $46 \%$, two-base substitutions at $31 \%$ and $18 \%$, and multiple substitutions at $7 \%$ and $2 \%$, for Regions I and V, respectively. These results suggest that primer annealing was adversely affected by even one mismatch and decreased substantially with each additional mismatch present.

\section{Mutagenesis Strategy for Central Region III}

Mutagenesis of regions at the ends of the element, such as I, II, V, and VI of $\mathrm{P}_{l y s}$ require only one PCR amplification with the opposing WT primer as diagrammed in Figure 1. Regions in the middle of the of the clones in Regions I and V, respec-

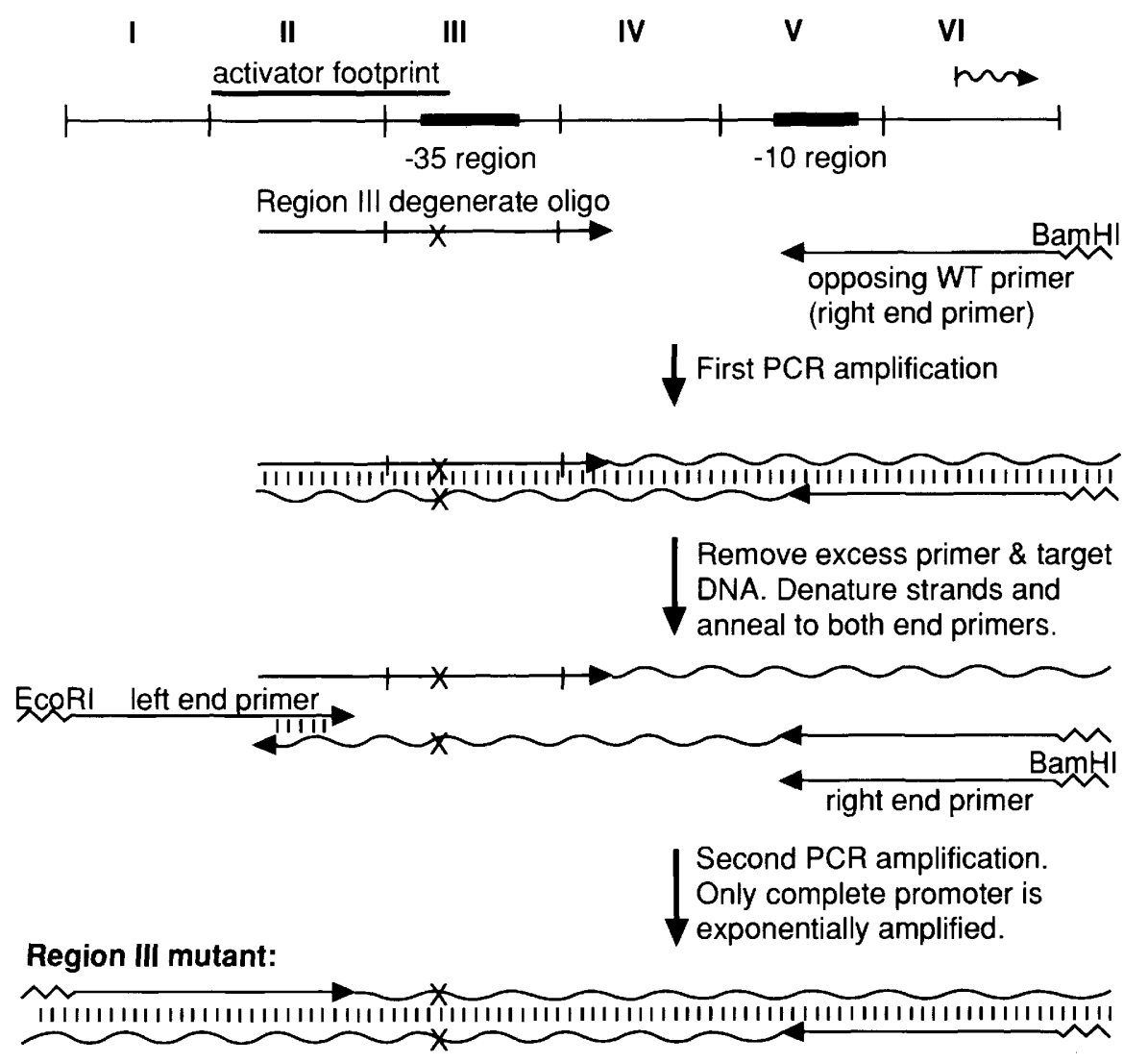

FIGURE 4 Recombinant PCR strategy to generate a Region III mutant population. 
element, such as III and IV, are not easily mutagenized with degenerate oligomers that extend to the ends of the element; not only does oligomer cost increase and yield decrease for longer oligomers, but the use of longer primers would be likely to increase the recovery of the less desirable multiple mutants. Therefore, mutagenesis of the central regions would be accomplished with a modified recombinant PCR method ${ }^{(5)}$ involving two sequential PCR amplifications. For example (see Fig. 4), in the first amplification, the opposing WT primer can be used with the degenerate primer to synthesize one end of the element. After gel purification of this short segment to remove excess primer and target DNA, the remaining end can be restored by adding back WT primers homologous to both ends of the element. The product generated from the first amplification would contain sequences overlapping with the WT primer from the missing end. Annealing, based on this homology would prime synthesis of the remaining base pairs during the second amplification. When this two-step recombinant PCR was applied to Regions III and IV, it effectively generated the desired mutant populations (data not shown). In cases where the element is considerably longer than $68 \mathrm{bp}$, multiple independent PCR products that overlap at their ends could be synthesized and combined with outside primers to generate the intact element.

\section{DISCUSSION}

The results presented here indicate that Mod-PCR is a convenient method for saturation mutagenesis of a DNA sequence element. Primer synthesis with the WT and appropriately diluted equimolar mixture of nucleotide substrates resulted in the generation of random base substitution mutations within the targeted region. Annealing conditions during ModPCR effectively reduced the recovery of mutants containing more than two base changes. Thus, Mod-PCR can be efficiently applied to independent saturation mutagenesis of distinct regions within a DNA sequence element, first defining the regions, and then the bases, important to element function.

Although not tested in this paper, we would presume that parameters affecting annealing conditions during PCR, such as temperature, $\mathrm{MgCl}_{2}$ concentration, and primer length could be altered to fine tune the distribution of single, double, and multiple base substitutions recovered. In addition, the recovery of WT clones could be reduced by increasing the rate of misincorporation during degenerate oligonucleotide synthesis, thereby reducing the proportion of WT primers. Table 3 illustrates the predicted effect of misincorporation rate on the isolation of specific classes of mutants for the Mod-PCR conditions used here. An increase in the average misincorporation rate from 1.5 to 2.5 misincorporations per primer would halve the number of WTs recovered in the Mod-PCR pool while increasing the number of double and multiple mutants. Because the frequency of single mutants would decrease significantly beyond 2.5 substitutions per primer, increased mutagenesis beyond 2.5 would be useful only when double and multiple mutants were also desirable. Alternatively, a colony hybridization assay ${ }^{(17)}$ with a WT primer as probe could be used to detect and eliminate WT clones from further analysis; for the rate of misincorporation tested $(0.15)$, this would have increased the proportion of single base substitutions to almost $70 \%$ of those sequenced.

Mod-PCR should provide an effective complement to the existing method of direct cloning of a degenerate oligonucleotide. ${ }^{(2-4)}$ Both methods take advantage of the ability to target mutations to a particular region through the use of synthetic degenerate oligonucleotides, and both can be used to generate a mutant pool containing random, predominantly single base mutations. The methods differ most significantly in the reduced cost of oligonucleotide synthesis for Mod-PCR and the absence of the need to introduce closely spaced restriction sites into the DNA being mutagenized by Mod-PCR. In its application to protein structure-function analysis, the direct cloning method has the advantage that use of a highly degenerate oligonucleotide can result in the recovery of many different amino acid changes at a single position, and the restriction sites needed for cloning can be introduced without changing the encoded amino acids. In contrast, such a change in a DNA sequence element may itself alter the activity of the element and affect the phenotypic properties of the targeted mutants, thereby resulting in erroneous conclusions. Lastly, the combination of Mod-PCR with recombinant PCR should allow the application of this method to saturation mutagenesis of intact wild-type DNA sequence elements too long to be mutagenized by the direct cloning method.

TABLE 3 Expected Effect of Misincorporation Rate During Mutagenic Oligonucleotide Synthesis on Mutant Recovery Following Mod-PCR

\begin{tabular}{|c|c|c|c|c|c|c|c|c|}
\hline \multirow[b]{2}{*}{ Rate $^{a}$} & \multicolumn{4}{|c|}{ Distribution in oligo pool ${ }^{\mathrm{b}}$} & \multicolumn{4}{|c|}{ Distribution in Mod-PCR pool ${ }^{\mathrm{c}}$} \\
\hline & 0 & 1 & 2 & more & 0 & 1 & 2 & more \\
\hline 0.100 & 0.31 & 0.38 & 0.21 & 0.10 & 0.54 & 0.36 & 0.09 & 0.01 \\
\hline 0.125 & 0.23 & 0.36 & 0.26 & 0.15 & 0.46 & 0.40 & 0.13 & 0.02 \\
\hline 0.129 & 0.22 & 0.36 & 0.26 & 0.16 & 0.45 & 0.40 & 0.14 & 0.02 \\
\hline 0.150 & 0.17 & 0.32 & 0.29 & 0.22 & 0.39 & 0.42 & 0.17 & 0.03 \\
\hline 0.175 & 0.12 & 0.28 & 0.30 & 0.30 & 0.33 & 0.42 & 0.20 & 0.04 \\
\hline 0.200 & 0.09 & 0.24 & 0.30 & 0.38 & 0.28 & 0.42 & 0.24 & 0.06 \\
\hline 0.225 & 0.06 & 0.20 & 0.28 & 0.46 & 0.23 & 0.41 & 0.27 & 0.09 \\
\hline 0.250 & 0.04 & 0.16 & 0.26 & 0.54 & 0.19 & 0.39 & 0.29 & 0.12 \\
\hline 0.275 & 0.03 & 0.12 & 0.23 & 0.62 & 0.16 & 0.36 & 0.31 & 0.17 \\
\hline 0.300 & 0.02 & 0.09 & 0.20 & 0.69 & 0.13 & 0.33 & 0.32 & 0.22 \\
\hline 0.325 & 0.01 & 0.07 & 0.17 & 0.75 & 0.10 & 0.29 & 0.32 & 0.28 \\
\hline 0.350 & 0.01 & 0.05 & 0.14 & 0.80 & 0.08 & 0.26 & 0.31 & 0.35 \\
\hline 0.375 & 0.01 & 0.04 & 0.11 & 0.84 & 0.06 & 0.22 & 0.29 & 0.43 \\
\hline
\end{tabular}

${ }^{a}$ Misincorporation rate during mutagenic oligonucleotide synthesis, adjusted by altering the concentration of nucleotide precursors in the equimolar mixture bottle.

${ }^{b}$ Determined by the binomial theorem for the indicated misincorporation rate and an 11-nucleotide region targeted for mutagenesis.

'Based on Mod-PCR conditions that result in 100\% recovery of the WT class, $55 \%$ recovery of the single mutant class, $25 \%$ for double mutants, and $5 \%$ for multiple mutants (average recovery rates observed for Regions I and V rounded to the nearest 5\%). 


\section{ACKNOWLEDGMENTS}

This work was supported by the College of Medicine, The University of Tennessee - Memphis, by National Science Foundation grant DMB 9006364 to M.M.H., and by a University of Tennessee Van Vleet Professorship. Oligonucleotides were provided by the Molecular Resource Center, The University of Tennessee - Memphis. The authors thank J. Swindle for advice on PCR and comments on the manuscript, and C.A. Baxa for providing template DNA and modified protocols for sequencing and chromosomal minipreps.

\section{REFERENCES}

1. Smith, M. 1985. In vitro mutagenesis. Annu. Rev. Genet. 19: 423-462.

2. Hill, D.E., A.R. Oliphant, and K. Struhl. 1986. Mutagenesis with degenerate oligonucleotides: An efficient method for saturating a defined DNA region with base pair substitutions. Methods Enzymol. 155: 558-568.

3. Bowie, J.U. and R.T. Sauer. 1989. Identifying determinants of folding and activity for a protein of unknown structure. Proc. Natl. Acad. Sci. USA. 86: 2152-2156.

4. Dube, D.K. and L.A. Loeb. 1989. Mutants generated by the insertion of random oligonucleotides into the active site of the $\beta$-lactamase gene. Biochemistry 28: 57035707.

5. Higuchi, R. 1989. Using PCR to engineer DNA. In PCR Technology (ed. H.A. Erlich), pp. 61-70. Stockton Press, New York.

6. Leung, E.W., E. Chen, and D.V. Goeddel. 1989. A method for random mutagenesis of a defined DNA segment using a modified polymerase chain reaction. Technique 1: 11-15.

7. Matteucci, M.D. and M.H. Caruthers. 1981. Synthesis of deoxynucleotides on a polymer support. J. Am. Chem. Soc. 103: 3185-3191.

8. Beaucage, S.L. and M.H. Caruthers. 1981. Deoxynucleoside phosphoramidites - a new class of intermediates for deoxypolynucleotide synthesis. Tetrahedron Lett. 22: 1859-1862.

9. Maniatis, T., E.F. Fritsch, and J. Sambrook. 1982. Molecular cloning: A laboratory manual. Cold Spring Harbor Press, Cold Spring Harbor, New York.

10. Grundy, F.J. and M.M. Howe. 1984. Involvement of the invertible $G$ segment in bacteriophage $\mathrm{Mu}$ tail fiber biosynthesis. Virology 134: 296-317.

11. Schleif, R.F. and P.C. Wensink. 1981. Practical methods in molecular biology. Springer-Verlag, New York.

12. Baxa, C.A., L. Chiang, and M.M. Howe.
1992. DNA sequence characterization of the $\mathrm{G}$ gene region of bacteriophage $\mathrm{Mu}$. DNA Sequence 2: 329-334.

13. Simons, R.W., F. Houman, and N. Kleckner. 1987. Improved single and multicopy lac-based cloning vectors for protein and operon fusions. Gene 53: 85-96.

14. Margolin, W., G. Rao, and M.M. Howe. 1989. Bacteriophage $\mathrm{Mu}$ late promoters: Four late transcripts initiate near a conserved sequence. J. Bacteriol. 171: 20032018.

15. Stark, M.J.R. 1987. Multicopy expression vectors carrying the $L a c$ repressor gene for regulated high-level expression of genes in Escherichia coli. Gene 51: 255-267.

16. Holmes, D.S. and M. Quigley. 1981. A rapid boiling method for the preparation of bacterial plasmids. Anal. Biochem. 114: 193-197.

17. Ausubel, F.M., R. Brent, R.E. Kingston, D.D. Moore, J.G. Seidman, J.A. Smith, and K. Struhl. 1991. Current protocols in molecular biology. John Wiley \& Sons, New York. 


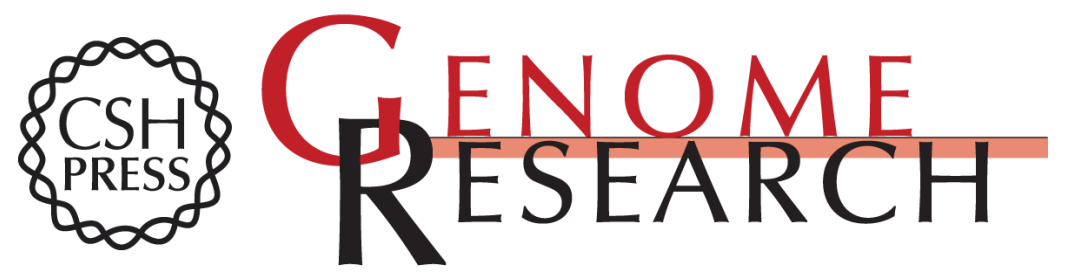

\section{Mutagenic oligonucleotide-directed PCR amplification (Mod-PCR): an efficient method for generating random base substitution mutations in a DNA sequence element.}

L W Chiang, I Kovari and M M Howe

Genome Res. 1993 2: 210-217

Access the most recent version at doi:10.1101/gr.2.3.210

References This article cites 13 articles, 2 of which can be accessed free at:

http://genome.cshlp.org/content/2/3/210.full.html\#ref-list-1

\section{License}

Email Alerting

Receive free email alerts when new articles cite this article - sign up in the box at the Service top right corner of the article or click here.

\section{Affordable, Accurate Sequencing.}

To subscribe to Genome Research go to:

https://genome.cshlp.org/subscriptions 